\title{
Políticas de la subjetividad en el régimen neoliberal. El "psicopoder" o la fábrica del homo consumens
}

\section{Politics of Subjectivity in the Neoliberal Regime. The "Psych Power" or the Homo Consumens' Factory}

\author{
Borja García Ferrer ${ }^{1}$ \\ Universidad Nacional Autónoma de México (México)
}

Recibido: 11-03-19

Aprobado: 09-07-19

\section{Resumen}

El principal cometido del presente trabajo es examinar el nivel productivoinfraestructural y subjetivo del régimen neoliberal, en contraste con la lógica puramente económica-empresarial del modo de producción capitalista en la era industrial y disciplinaria. En primer lugar, examinamos la noción foucaultiana de "biopolítica" y su recepción en el pensamiento de Deleuze, analizando el papel pretendidamente esencial que juegan los deseos humanos para el funcionamiento del capitalismo de consumo. A la luz de las averiguaciones logradas, indagamos en perspectiva histórica las principales tecnologías donde se cifra la eficacia del "psicopoder" emergente, con el objeto de dilucidar, a la luz de las nuevas formas de producción inmateriales y en red, su sentido y sus efectos en las subjetividades y las formas de vida.

Palabras-clave: "Psicopoder", neoliberalismo, consumismo, deseo, Big Data.

\footnotetext{
${ }^{1}$ (borja_co@hotmail.com) Doctor en Filosofía por la Universidad de Granada. Actualmente ejerce como investigador postdoctoral en la Facultad de Filosofía y Letras de la Universidad Nacional Autónoma de México. Sus intereses de investigación actuales son la filosofía contemporánea, la ontología política, la sociología crítica y la psicopatología. Entre sus publicaciones cabe señalar "La constitución de la identidad personal en el nuevo capitalismo y sus repercusiones morales", Daimon. Revista Internacional de Filosofia, 67 (2016), pp. 117- 131. "Revolución espacial y 'desarraigo'. Una crítica de sus implicaciones psico-somáticas en el capitalismo virtual”, Pensamiento. Revista de Investigación e Información Filosófica, 72, 274 (2016), pp. 1175-1196. "La 'agenesia' como patología de civilización y su alcance en el capitalismo de la flexibilidad”, Co-herencia, 14, 26 (2017), pp. 119150. En Araucaria publicó "La 'dromocracia' o el régimen de la velocidad absoluta (Paul Virilio). Un diagnóstico de sus derivaciones mórbidas en la existencia”, 19, 38, 2017, pp. 49-71.
}

ORCID: http://orcid.org/0000-0001-6585-4857. 


\begin{abstract}
The main task of this paper is to examine the productive-infrastructural and subjective level of the neoliberal regime, in contrast to the purely economic-business logic of the capitalist mode of production in the industrial and disciplinary era. First, we examine the Foucauldian notion of "biopolitics" and its reception in Deleuze's thought, analyzing the supposedly essential role played by human desires for the functioning of consumer capitalism. In the light of the inquiries made, we investigate in historical perspective the main technologies where the effectiveness of the emerging "psych power" is calculated, with the purpose of elucidating, in the light of the new immaterial and networked forms of production, its meaning and its effects on subjectivities and life forms.
\end{abstract}

Key-words: "Psych power", neoliberalism, consumerism, wish, Big Data.

Lo real no es imposible, es cada vez más artificial G. Deleuze y F. Guattari, El Anti Edipo

\title{
1. Introducción
}

Una vez consumado el proceso conocido genéricamente como "Revolución Industrial", numerosos intelectuales de las más diversas disciplinas han denunciado bajo diversos puntos de vista las consecuencias nocivas del modo de producción capitalista para los seres humanos. En esta tradición de pensamiento, brilla con luz propia la figura de K. Marx, intérprete aventajado de Hegel y cabeza visible de una corriente filosófica que persiste e insiste con inusitada vehemencia en el presente inmediato. Como es sabido, el filósofo alemán imputa los efectos de "alienación", precariedad y desigualdad que comporta el capitalismo industrial de la época victoriana en aras de la producción y del beneficio económico. Marx denomina "acumulación originaria" al proceso violento donde se cifra el tránsito del modo de producción feudal al modo de producción capitalista, sustrayendo los medios de producción a los productores y generando, de este modo, las condiciones materiales necesarias para el funcionamiento del capitalismo. Ahora bien, la "acumulación originaria" no redunda exclusivamente en la desposesión, la rapiña y la acumulación de bienes porque, para ser capital, la riqueza requiere unas condiciones sociales muy determinadas. Además de sustraer las propiedades comunes, la "acumulación originaria" supone grandes dosis de violencia en las políticas dirigidas a la producción del trabajador asalariado, una violencia que ha dejado su huella en la historia, en palabras de Marx, con "trazos indelebles de 
sangre y fuego"2. Desigual y jerarquizada, la división social del trabajo somete al proletariado a unas condiciones de trabajo deshumanizantes; unas condiciones que "engendran, garantizan y perpetúan"3 el predominio del capital.

No nos pasa desapercibido que, aun con sus especificidades, los dispositivos y mecanismos descritos por Marx siguen teniendo vigencia en la periferia del mundo, pero también en los países con un capitalismo más "avanzado" (organización científica del trabajo, modo de producción en serie, maquinización, políticas extractivas, coacción al trabajo, precarización, etc.). Más allá de toda lectura reduccionista, el nuevo capitalismo se define, de hecho, como una especie de exposición universal de los modelos productivos ${ }^{4}$ que tiene a la violencia extraeconómica como único denominador común. A pesar de Marx, la "acumulación originaria" no constituye un fenómeno espaciotemporal limitado a la prehistoria de la era burguesa: lejos de limitarse a propiciar su gestación y posterior desempeño, designa un proceso coextensivo al modo de producción capitalista, con independencia de sus diferentes episodios históricos ${ }^{5}$. Según nuestra hipótesis, empero, la vertiente neoliberal del capitalismo trae consigo una diferencia cualitativa en el modo de implementar la "acumulación originaria", una forma intensiva que no solo concierne al espacio exterior del planeta, como denota el fenómeno de la globalización, sino que se proyecta asimismo sobre nuestro espacio interior, erigiendo la producción del sujeto como el eje subjetivo de una nueva "acumulación originaria". En efecto, si el "trabajo consumidor" (consumption work) es una categoría fundamental para entender nuestra época ${ }^{6}$, es porque la lógica de dominación capitalista trasciende la esfera laboral hasta colonizar la propia vida, convirtiendo la especie humana en un rebaño de consumidores voraces e insaciables. De tal suerte que el capitalismo no es simplemente un proyecto de explotación, como pretende la escolástica marxista; en última instancia, se constituye como una fábrica de subjetividades, y su principal cometido se cifra en la producción y desinhibición de nuestros deseos. Para decirlo con la fórmula de Boltanski y Chiapello, el "nuevo espíritu del capitalismo" tiene una dimensión antropogenética fundamental; en términos de Lazzarato: "Aquello que definimos como 'economía' sería lisa y llanamente imposible sin la producción y el control de la subjetividad y de sus formas de vida"”.

\footnotetext{
${ }^{2}$ Karl Marx, El Capital, México, Siglo XXI, 1975, p. 894.

${ }^{3}$ Ibid., p. 922.

${ }^{4}$ Cfr. Paolo Virno, Gramática de la multitud: para un análisis de las formas de vida contemporáneas, Madrid, Traficantes de sueños, 2003.

${ }^{5}$ En este sentido, el geógrafo británico D. Harvey prefiere utilizar la fórmula "acumulación por desposesión", en lugar de "acumulación originaria". Cfr. David Harvey, Guía de El Capital de Marx. Libro Primero, Madrid, Akal, 2014.

${ }^{6}$ Cfr. Kathryn Wheeler y Miriam Glucksmann, Household recycling and consumption work: social and moral economies, Londres, Palgrave McMillan, 2015.

' Maurizio Lazzarato, La fábrica del hombre endeudado. Ensayo sobre la condición neoliberal, Buenos Aires, Amorrortu, 2013, p. 41.
}

Araucaria. Revista Iberoamericana de Filosofia, Política, Humanidades y Relaciones Internacionales, año $22, \mathrm{n}^{\circ} 43$. Primer semestre de 2020. Pp. 55-76. ISSN 1575-6823 e-ISSN 2340-2199 https://dx.doi.org/10.12795/araucaria.2020.i43.03 


\section{De la "sociedad disciplinaria" ("biopoder") a la "sociedad de control" ("psicopoder")}

Para mostrar el sentido de nuestra hipótesis y probar su impacto en los sujetos de carne y hueso, se impone la necesidad de realizar, como diría Marx, un "plusvalor" hermenéutico del concepto foucaultiano de "biopolítica". Y es que, si bien es verdad que la cuestión de la "biopolítica" protagoniza muchos de los debates en torno al "nuevo espíritu del capitalismo", no es menos cierto que la categoría en cuestión se caracteriza por una gran incertidumbre semántica, lo cual se traduce con demasiada frecuencia en interpretaciones extrañas e, incluso, opuestas entre $\mathrm{si}^{8}$.

Desde nuestro punto de vista, el capitalismo industrial es uno de los rostros (quizás, el más representativo) de una realidad pretendidamente omniabarcante, holística y poliédrica: la "sociedad disciplinaria". Como consecuencia del giro de la forma de producción agraria a la industrial, la naturaleza del poder experimenta en el siglo XVII una metamorfosis radical: concebido tradicionalmente como un poder de muerte, el poder "soberano" se trueca en "disciplinario" o "biopolítico", en el sentido de que su cometido ya no sería matar, sino la "administración de los cuerpos" y la "gestión calculadora de la vida". Se trata, aclara Foucault, de una "ortopedia concertada" minucioso y una coacción calculada para disciplinar el cuerpo en términos de docilidad-utilidad, o sea, sujetar constantemente sus fuerzas y ajustarlas con rutinaria precisión a los ritmos marcados por la "maquinaria" (Machenschaft) ${ }^{11}$. En suma, la "biopolítica disciplinaria" se define esencialmente como una forma de gobierno normativo, mientras que su principal resultado sería la construcción del sujeto como una máquina de producción obediente y eficiente al mismo tiempo.

En tal disposición de los términos, Foucault identifica el panopticon de Bentham como el modelo generalizable de la vigilancia moderna, un modelo que conecta inextricablemente las relaciones de poder con la vida cotidiana de los hombres ${ }^{12}$. Como síntesis de la sociedad disciplinaria, el panóptico se fundamenta en una relación de dependencia entre vigilantes y vigilados (en el caso concreto que nos ocupa, la burguesía y el proletariado), en el marco de un espacio fijo y cerrado sobre sí mismo en todos sus puntos (la fábrica) donde todo movimiento y todo acontecimiento vienen inmediatamente controlados

\footnotetext{
${ }^{8}$ Cfr. Roberto Esposito, Bíos. Biopolitica y filosofia, Buenos Aires, Amorrortu, 2006.

9 Cfr. Michel Foucault, Historia de la sexualidad I. La voluntad de saber, Madrid, Siglo XXI, 2006, pp. 166-167.

10 Ibid., p. 169.

${ }^{11}$ Cfr. Michel Foucault, Vigilar y castigar. Nacimiento de la prisión, Buenos Aires, Siglo XXI, 2002, p. 126.

${ }^{12}$ Cfr. Ibid., p. 208.
} 
y registrados como conditio sine qua non para normalizar y encauzar la conducta. A tenor del criterio seguido en cada caso (obediencia, productividad, eficiencia, utilidad, etc.), el panóptico localiza y clasifica exhaustivamente a los individuos que trabajan o conviven juntos, instaurando de este modo el orden social. Distribuidos y ordenados en el espacio-tiempo, individualizados y separados, los objetos de la vigilancia se ven impelidos a asumir las normas prescritas por sus vigilantes como propias, bajo la certeza de saberse sometidos constantemente al más riguroso escrutinio. Así pues, el panóptico se mantiene siempre visible y, al mismo tiempo, inverificable, garantizando el funcionamiento automático del poder en el sistema donde se aplique ${ }^{13}$.

En otro orden de cosas, Marx presenta el capitalismo en su perspicaz análisis del proceso de transformación del dinero en capital como el reino de lo ilimitado por excelencia, en virtud de lo cual le atribuye una naturaleza más metafísica que económica: "La circulación del dinero como capital es [...] un fin en sí, pues la valorización del valor existe únicamente en el marco de este movimiento renovado sin cesar. El movimiento del capital [...] es carente de medida"14. Desde este prisma, para nosotros, el principio de "acumulación originaria" tiene como sustrato y soporte metafísico el concepto nietzscheano de "voluntad". En su particular deconstrucción de la historia de la metafísica (esto es, del "olvido del ser" o "nihilismo impropio"), Heidegger advierte que la noción de "voluntad de poder" (Der Wille zur Macht) reduce el ser a valor hasta convertirlo, parafraseando al mismo Nietzsche cuando critica, curiosamente, el concepto de "ser", en el "último humo de la realidad que se evapora" 15 . En otras palabras, Nietzsche llevaría hasta el extremo, a juicio de Heidegger, la metafísica de la subjetividad (del ego cogito al ego volo), de tal suerte que su pensamiento "ha recorrido el círculo de las posibilidades que le estaban señaladas de antemano"16. Una vez consumada la metafísica, sus principios fundamentales se encarnan de forma ineluctable en la estructura material de la realidad efectiva, propiciando la instalación manipulable de un mundo científico-técnico. En este contexto, la idea metafísica de "voluntad" se torna "voluntad de voluntad", a saber, la característica principal de la técnica a nivel planetario. Se trata de la producción absoluta de la totalidad del ente por la sola voluntad, en el sentido de un insaciable "producir por producir"

13 Cfr. Ibid., p. 204.

14 Karl Marx, El Capital, op. cit., p. 186. Marx tematiza la diferencia fundamental entre el mundo precapitalista y el capitalista sobre la base implícita de la contraposición aristotélica entre economía y crematística. Cfr. Aristóteles, De Republica, Buenos Aires, Losada, 2005, libro I, caps. 8 y 9 et passim.

${ }^{15}$ Friedrich Nietzsche, Crepúsculo de los ídolos, Madrid, Alianza, 1980, p. 47.

16 Martin Heidegger, "La superación de la metafísica" [en Eustaquio Barjau, trad.: Conferencias y artículos, Barcelona, Serbal, 1994], p. 60. Hemos profundizado sobre esta problemática en: Borja García Ferrer, "A vueltas con Heidegger. La filosofía como terapia frente al "nihilismo tecnológico"" [en Luis Sáez Rueda, ed.: El malestar de Occidente. Perspectivas filosóficas sobre una civilización enferma, Anchor Academic Publishing, Hamburg, 2017], pp. 33-36.

Araucaria. Revista Iberoamericana de Filosofia, Politica, Humanidades y Relaciones Internacionales, año $22, \mathrm{n}^{\circ} 43$. Primer semestre de 2020. Pp. 55-76. ISSN 1575-6823 e-ISSN 2340-2199 https://dx.doi.org/10.12795/araucaria.2020.i43.03 
(más allá de las necesidades concretas) ${ }^{17}$. Pues bien, desde nuestro punto de vista, es factible aplicar la lectura heideggeriana de la técnica al capital. Para nosotros, la "acumulación originaria" es un principio tautológico, en el sentido de que, por mor del afán productivista del capitalismo, solo aspira a su propio perfeccionamiento, sin ninguna finalidad externa. Hablando con Hegel, el "mal infinito" designa la secreta teleología del capital, esto es, la producción desmesurada como fin en sí mismo. Como la técnica de Heidegger, es un modo de producción autonomizado e ilimitado por definición ${ }^{18}$.

Vistas las cosas así, el dispositivo opaco-transparente del panóptico se revela, ciertamente, como una técnica de poder demasiado restringida ${ }^{19}$. Tal y como ha sido descrito hasta ahora, todo hace indicar que nos encontramos ante un dispositivo de vigilancia eminentemente fuerte ${ }^{20}$. Bien pensado, empero, no se trata de un dispositivo tan robusto como cabría pensar a ojos vista, ya que su código de normas, preceptos y prohibiciones no puede abarcarlo todo. En efecto, la restricción de los campos de visión en los espacios cerrados que lo conforman permite dibujar líneas de fuga en la exterioridad de la esfera laboral, allí donde los sujetos, lejos del alcance de la vigilancia, pueden dar rienda suelta a sus necesidades, sus anhelos ocultos y sus deseos, actuar libremente y ser lo que quieran ${ }^{21}$. Sirvan como botón de muestra las condiciones de sociabilidad del proletariado en el mundo histórico de Marx: en la medida en que sus viviendas se hallaban, en la gran mayoría de casos, en condiciones deplorables, la vida ordinaria de los obreros discurría fundamentalmente en la calle y en la taberna, conocida elocuentemente en las corrientes socialistas como "salón del obrero"; pues bien, concebidas como "redes sumergidas" en la vida cotidiana ${ }^{22}$, tanto la una como la otra permitían transmitir experiencias comunes, construir solidaridades, identidades comunitarias y, a la postre, conspirar contra los empresarios en la cotidianeidad de la vida privada y del tiempo libre. Más recientemente, numerosos acontecimientos históricos atestiguan las limitaciones espaciales del panóptico, entre los cuales cabe destacar Mayo del 68 y sus diversas formas de resistencia colectiva al modelo de producción y acumulación disciplinario bajo la bandera de la imaginación y

${ }^{17}$ Cfr. Diego Sánchez Meca, El nihilismo. Perspectivas sobre la historia espiritual de Europa, Madrid, Síntesis, 2004, p. 181 ss.

${ }^{18}$ Heidegger llama "usura" al funcionamiento de la técnica en cuanto tal, sin ninguna meta que oriente su decurso. Cfr. Martin Heidegger, op. cit., p. 67.

${ }^{19}$ Deleuze diagnostica la crisis general de los entornos de reclusión, firmando una de las páginas doradas de la filosofía contemporánea. Cfr. Gilles Deleuze, "Post-scriptum sobre las sociedades de control" [en Conversaciones 1972-1990, Valencia, Pre-Textos, 1999], p. 278.

${ }^{20}$ Cfr. David Lyon, "The search for surveillance theroies" [en Theorizing surveillance: the panopticon and beyond, Cullompton, Willan Publishing, 2006], pp. 3-20.

${ }^{21}$ Cfr. William Bogard, "Surveillance assemblages and lines of flight" [en Theorizing surveillance: the panopticon and beyond, Cullompton, Willan Publishing, 2006], pp. 97-122.

${ }_{22}$ Alberto Melucci, Acción colectiva, vida cotidiana y democracia, Ciudad de México, Colegio de México, 1999.

Araucaria. Revista Iberoamericana de Filosofia, Política, Humanidades y Relaciones Internacionales, año $22, \mathrm{n}^{\circ} 43$. Primer semestre de 2020. Pp. 55-76. ISSN 1575-6823 e-ISSN 2340-2199 https://dx.doi.org/10.12795/araucaria.2020.i43.03 
la libertad sexual ${ }^{23}$. Y es que, como señala uno de sus grandes gurús filosóficos, H. Marcuse, si bien son necesarias ciertas dosis de opresión y restricciones de la libertad para el desarrollo de la civilización, la negatividad del adiestramiento y la represión disciplinaria del "principio de placer" (Freud) en la sociedad industrial resultan a todas luces insostenibles ${ }^{24}$.

Por otra parte, existen evidencias empíricas de que el capital no puede superar, en cuanto poder panóptico, un determinado nivel de productividad. Por oposición a la célebre tesis de Marx sobre la necesaria extinción del capitalismo por mor de un decrecimiento inexorable de la "tasa de ganancia" (dicho con su famoso apotegma, "la burguesía produce sus propios sepultureros"), lo cierto es que el capital ha visto incrementado progresivamente su rendimiento (con la única excepción de los períodos correspondientes a las dos Guerras Mundiales) a lo largo del siglo XX, como pone de manifiesto Th. Piketty en su minucioso análisis histórico de los distintos procesos de acumulación de riqueza en las principales economías ${ }^{25}$. Esta bonanza económica tuvo su punto más álgido, como se sabe, durante los treinta años sucesivos a la II Guerra Mundial, conocidos como la "Edad de oro del capitalismo" (Trente Glorieuses o "Treinta Gloriosos" en Francia, Nachkriegsboom o "boom de la posguerra" en Alemania). Derrumbada la fe otrora inquebrantable en la mano invisible del mercado, el éxito del capitalismo en este período se debe en buena medida a los esfuerzos del denominado "socialismo liberal" (J. M. Keynes, L. Hobhouse, etc.) por refundar el liberalismo mediante el fortalecimiento de la función reguladora y distributiva del Estado, a fin de implementar una libertad no solo "formal", sino también (y sobre todo) "real". Sin embargo, la crisis del petróleo de 1973 tuvo como corolario imprevisto un estancamiento de la producción sin precedentes, una crisis de legitimidad que ponía en jaque el modelo fordista a nivel productivo, pero también el modelo del socialismo liberal en el terreno de la circulación ${ }^{26}$.

Acuciado por semejante crisis de legitimidad, el gran reto del capitalismo no era sino superar las limitaciones y restricciones del panóptico disciplinario, sus espacios preconstruidos y sus técnicas ortopédicas para satisfacer el apetito

${ }^{23}$ No obstante, es de recibo matizar que Mayo del 68 se basó como uno de sus pilares fundamentales en un mecanismo estrechamente vinculado a las formas de trabajo disciplinadas que caracterizan al modo de regulación keynesiano-fordista como es la huelga. Cfr. Sergio Bologna y Giairo Daghini, Maggio '68 in Francia, Roma, DeriveApprodi, 2008. Cfr. Bruno Astarian, Las huelgas en Francia durante mayo y junio de 1968, Madrid, Traficantes de Sueños, 2008.

${ }^{24}$ Cfr. Herbert Marcuse, Eros y Civilización, Barcelona, Seix Barral, 1976.

${ }_{25}$ Cfr. Thomas Pikkety, El capital en el siglo XXI, Madrid, Fondo de Cultura Económica, 2014.

26 Junto a los límites del modelo fordista de producción, es de recibo señalar que hubo otras dimensiones complementarias de semejante crisis de legitimidad del sistema capitalista, como la incapacidad de los Estados para continuar fungiendo como avales últimos ante la extraordinaria envergadura alcanzada por la deuda pública, o aspectos que no están necesariamente relacionados con la dimensión disciplinaria del trabajo, como la gigantificación de las empresas o los cambios en la política de importaciones en los países de la periferia capitalista.

Araucaria. Revista Iberoamericana de Filosofía, Política, Humanidades y Relaciones Internacionales, año $22, \mathrm{n}^{\circ} 43$. Primer semestre de 2020. Pp. 55-76. ISSN 1575-6823 e-ISSN 2340-2199 https://dx.doi.org/10.12795/araucaria.2020.i43.03 
insaciable que lo caracteriza, ya sea mediante la apropiación de recursos y de fuerza de trabajo más eficientes, o bien extendiendo las relaciones capitalistas a una escala mucho más vasta. Pues bien, probando nuevamente su capacidad para superar cualquier obstáculo, el modo de producción capitalista opera a mediados del siglo XX una auténtica revolución hacia una mayor apertura y deslimitación; no obstante, esta mutación no debe ser entendida como la irrupción de un sistema completamente nuevo sino como una optimización y una exacerbación del capitalismo mismo, concebidas indistintamente para aumentar la productividad ad infinitum.

En sus conocidas reflexiones sobre el imperialismo, R. Luxemburgo subraya que la lógica de funcionamiento del capital encierra una necesidad inmanente, empujada por crisis estructurales; se trata de la necesidad de superar incesantemente sus límites, incorporando siempre nuevos espacios. Así, a ojos de la autora, el cometido del colonialismo no sería otro que someter mediante el uso de la violencia a territorios no capitalistas, reproduciendo a gran escala el principio marxista de la "acumulación originaria" 27 . Desde este punto de vista, es de recibo reconocer a $\mathrm{W}$. Lippmann el mérito incuestionable de representar el neoliberalismo como una práctica gubernamental dirigida a la producción de un homo oeconomicus neoliberal, en lugar de como una teoría política o económica (en el sentido de Marx). En contraste con la propuesta de Keynes, Hobhouse y compañía para refundar el liberalismo, el intelectual americano advierte en el Coloquio Walter Lippmann de $1938^{28}$ que el neoliberalismo no pretende implantar un modelo ideal, como demuestra la gran diversidad de experiencias históricas neoliberales; bien pensado, su principal cometido es adecuar la "sociedad humana a la mutación industrial y comercial"22. Así pues, la política neoliberal debe modificar al ser humano, entendido como una suerte de "inadaptado crónico", transformando el modo en que se representa su vida y su destino ${ }^{30}$. Es lo que Foucault elucubrará años más tarde, en los cursos del Collège de France, como "gubernamentalidad neoliberal". Sin embargo, los análisis del neoliberalismo a cargo del filósofo francés permanecen anclados en un esquema, el disciplinario, incapaz de vislumbrar la verdadera piedra filosofal de la "nueva razón del mundo", como reza la célebre obra de Laval y Dardot. El problema radica, a nuestro juicio, en que dicho esquema no hace justicia

${ }^{27}$ Cfr. Rosa Luxemburgo, La acumulación del capital, Madrid, Ediciones Internacionales Sedov, 2011. S. Amin extrapola la perspectiva marxiana de Luxemburgo a la lógica geopolítica que acrecienta progresivamente la desigualdad entre el centro y la periferia del mundo. Cfr. Samir Amin, La acumulación en escala mundial, Buenos Aires, Siglo XXI, 1975.

${ }^{28}$ Esta sede constituye un hito fundamental en la historia del liberalismo, hasta el punto de ser considerada como una especie de "Internacional liberal". Cfr. Christian Laval y Pierre Dardot, $L a$ nueva razón del mundo. Ensayo sobre la sociedad neoliberal, Barcelona, Gedisa, 2013, pp. 67-97.

29 Walter Lippmann, The Good Society, Boston, Little, Brown and Company, 1937.

${ }^{30}$ Cfr. Andrea Fagioli, “Acumulación originaria y capitalismo neoliberal. Una posible lectura del Chile post-golpe”, en Isegoría. Revista de Filosofia Moral y Politica, 59 (2018), p. 582. 
al tránsito infraestructural-productivo y subjetivo que supone la expansión neoliberal del dominio capitalista al "mundo de la vida" (Lebenswelt).

A nuestro juicio, si el principal cometido del capitalismo industrial era producir disciplinados poseedores de fuerza de trabajo en el ámbito de la fábrica, el capitalismo neoliberal se vuelca esencialmente en la vida emocional, es decir, en la gestión de nuestras pasiones y nuestros sentimientos más íntimos, confinados hasta entonces en los márgenes de la vida laboral. Y es que, para incrementar la productividad (orientada sobre todo al consumo, pero también al trabajo), es a todas luces necesario "intervenir la psique y condicionarla a un nivel pre-reflexivo" ${ }^{31}$. Se entiende, desde esta perspectiva, la célebre misiva lanzada por M. Thatcher a la posteridad, según la cual la economía no sería más que un método cuyo verdadero cometido se trataría de cambiar corazones y $\operatorname{almas}^{32}$. Como dice Deleuze, mientras que el régimen disciplinario se organiza como un "cuerpo", el régimen neoliberal hace lo propio como "alma"33, determinando de este modo su condición y su materia. Hemos pasado del "amaestramiento de los cuerpos" a la "gestión de los espíritus". De aquí que la "psicopolítica" (apostilla Byung-Chul Han) se imponga progresivamente como la forma de gobierno por antonomasia ${ }^{34}$.

Así entendido, el capitalismo aparece, siguiendo a Deleuze, como una "máquina demente" sin antecedentes históricos. Para el pensador francés, las sociedades preexistentes al capitalismo (tanto las antiguas máquinas territoriales como las sucesivas máquinas despóticas) tienen un denominador común: todas ellas operan en base a flujos codificados. Desde este prisma, el principal cometido de toda máquina social ha sido desde siempre impedir que fluyan flujos sobre su cuerpo que se resistan a ser codificados. De hecho, los flujos descodificados han tenido invariablemente un efecto de pánico en la sociedad, en la medida en que pueden disolver fácilmente la tierra que habitan. En el momento en que algo aparecía como incodificable, el aparato represivo ha utilizado todos los medios a su alcance para aniquilarlo y, en segunda instancia, recodificarlo o, mejor dicho, sobrecodificarlo. Por tanto, la principal función de la sociedad ha sido tradicionalmente codificar los flujos y tratar como enemigo todo lo que se presente, en relación a ella, como un flujo no codificable. Pues bien, en opinión de Deleuze, el modo de producción capitalista encierra una paradoja fundamental: si los flujos descodificados han significado el terror para todas las formaciones sociales, el capitalismo se funda sobre la quiebra de todos los códigos y, más precisamente, en el encuentro entre flujos deseantes

\footnotetext{
${ }^{31}$ Byung-Chul Han, Psicopolitica. Neoliberalismo y nuevas técnicas de poder, Barcelona, Herder, 2014, p. 33. 1981.

${ }^{32}$ Cfr. Ronald Butt, "Mrs Thatcher: The First Two Years", en The Sunday Times, 3 de mayo de

${ }^{33}$ Cfr. Gilles Deleuze, op. cit., p. 279.

${ }^{34}$ Cfr. Byung-Chul Han, Psicopolítica ..., op. cit., p. 33.
}

Araucaria. Revista Iberoamericana de Filosofia, Política, Humanidades y Relaciones Internacionales, año $22, \mathrm{n}^{\circ} 43$. Primer semestre de 2020. Pp. 55-76. ISSN 1575-6823 e-ISSN 2340-2199 https://dx.doi.org/10.12795/araucaria.2020.i43.03 
de cualquier naturaleza; de tal suerte que lo que era esencialmente negativo ha devenido la positividad misma de nuestra formación.

Llegados a este punto, la pregunta es, ¿cómo puede funcionar, bajo qué forma, una sociedad que, por oposición a todas las sociedades precedentes, gira en torno a flujos deseantes? Como afirma Deleuze en la senda de Nietzsche, la tarea del filósofo no se limita a reproducir lo que dicen sus maestros o una determinada corriente de pensamiento; antes bien, su razón de ser estriba en crear nuevos conceptos para problemas que cambian necesariamente, tal y como determina la tesis marxista del "materialismo histórico". Pues bien, esto es precisamente lo que hace el filósofo francés de la diferencia: sus principales nociones políticas siguen la estela de Marx o Foucault, pero adaptándose con especial clarividencia a las condiciones impuestas por el capitalismo neoliberal. La clave de la interrogante que nos ocupa radica, según Deleuze, en el fundamento de la relación entre capitalismo y esquizofrenia, esto es, lo que tienen completamente en común, su profunda identidad. Contra la creencia más arraigada en la tradición psicopatológica ${ }^{35}$, la esquizofrenia no designa simplemente la afección del esquizofrénico artificializado y personificado en el autismo. En realidad, no existe algo parecido a una especificidad o entidad esquizofrénica. La esquizofrenia tampoco es un modo de vida o una visión del mundo. Bien pensado, se trata de un proceso de producción, la universal producción primaria como "realidad esencial del hombre y de la naturaleza"36. Más precisamente, es el proceso de la producción del deseo y de máquinas deseantes, un proceso entendido, en la línea de Marx, como realidad material económica, como "proceso de producción en la identidad Naturaleza = Industria"37. La naturaleza, por tanto, en cuanto producción o industria, producción del hombre y por el hombre.

Ahora bien, ¿qué entiende Deleuze por deseo? Bajo su punto de vista, el capitalismo produce el deseo como carencia, en detrimento de su poder interior para engendrar su objeto ${ }^{38}$. Para Deleuze, el deseo se define como el conjunto de síntesis pasivas que maquinan los objetos parciales, los flujos y los cuerpos, síntesis pasivas que tienen por objeto la producción de realidad. El ser objetivo del deseo es, por tanto, lo real en sí mismo: desear es producir, producir en realidad. Desde este prisma, el deseo apenas tiene necesidades; al contrario, son las necesidades las que se derivan del deseo. Pues bien, siguiendo a Deleuze, el capitalismo inscribe el deseo en la lógica de la adquisición, de manera tal que lo destituye de su ser objetivo. Para ello, extrae hasta las últimas consecuencias de la concepción clásica o idealista (dialéctica y nihilista) del deseo que subyace

\footnotetext{
35 Cfr. Gilles Deleuze y Félix Guattari, El Anti Edipo. Capitalismo y esquizofrenia, Barcelona, Paidós, 1985, p. 30.

${ }^{36}$ Ibid., p. 14.

${ }^{37}$ Ibid., p. 40.

${ }^{38}$ Cfr. Ibid., p. 32 ss.
}

Araucaria. Revista Iberoamericana de Filosofia, Política, Humanidades y Relaciones Internacionales, año $22, \mathrm{n}^{\circ} 43$. Primer semestre de 2020. Pp. 55-76. ISSN 1575-6823 e-ISSN 2340-2199 https://dx.doi.org/10.12795/araucaria.2020.i43.03 
a la tradición psicoanalítica, también conocida como "teoría del apoyo" o "anaclisis". Desde la óptica del psicoanálisis, el deseo es concebido como una clase de producción muy determinada, a saber, como producción de fantasmas. Esto significa que el objeto real del que carece el deseo remite a una producción natural o social extrínseca, mientras que el deseo produce el fantasma y se produce a sí mismo, distanciándose del objeto y llevando la carencia, como consecuencia, hasta el extremo de convertirla en una "incurable insuficiencia de ser".

Así pues, el psicoanálisis concibe el deseo como una realidad psíquica (fantasmática $u$ onírica), en contraposición a la realidad material de la producción social. Sin embargo, Deleuze refuta esta teoría porque no existe una producción social de realidad, por un lado, y una producción deseante de fantasmas, por otro. Lo que acontece es una represión social sobre la producción deseante, pero esto no cambia en absoluto el principio de que el deseo produce lo real. A decir verdad, los fantasmas no son más que expresiones secundarias: no existe nada más que el deseo y lo social. Por otro lado, la diferencia que media entre las máquinas deseantes y las máquinas sociales o técnicas no es una diferencia de naturaleza sino de régimen (según relaciones de tamaño), o sea, las máquinas deseantes son al mismo tiempo técnicas y sociales. La producción social es solo la producción deseante en condiciones determinadas (en nuestro caso, atravesada por la carencia), es decir, los flujos deseantes que recorren el campo social son productos determinados históricamente. En palabras del propio autor:

"La carencia es preparada, organizada, en la producción social, [la cual] se vuelca sobre las fuerzas productivas y se las apropia. [...] La producción nunca es organizada en función de una escasez anterior, es la escasez la que se aloja [y] se propaga según la organización de una producción previa. Es el arte de una clase dominante, práctica del vacío como economía de mercado: organizar la escasez, la carencia, en la abundancia de producción"39.

Desde esta perspectiva, Deleuze analiza el proceso de producción deseante y advierte que la identidad entre capitalismo y esquizofrenia hunde sus raíces en la comunidad de un principio abstracto, a saber, emitir, hacer pasar, interceptar y concentrar los flujos descodificados. En cuanto máquina esquizofrénica, el capitalismo actúa como un aparato de captura de todos los flujos deseantes que recorren la sociedad, en virtud de una micropolítica que pone en marcha un doble movimiento: por un lado, descodifica las codificaciones cualitativas del deseo $\mathrm{y}$, al mismo tiempo, las recodifica mediante la apropiación privada de la "plusvalía"40. Así pues, capitalismo

${ }^{39}$ Ibid., p. 35.

40 Deleuze y Guattari alinean el doble movimiento de la descodificación y la recodificación 
como descodificación y como conjunción de los flujos descodificados. La descodificación esquizofrénica se ve doblada por la recodificación paranoica. Esquizofrenia y paranoia. La primera deshace los flujos de deseo y la segunda los reconstruye, constituyéndose como un potente motor de diferencias insertadas en modelos de identidad estándar ${ }^{41}$. En este sentido, el capitalismo no deja de contrariar su tendencia más esencial al mismo tiempo que se precipita sobre ella: si bien tiende a un umbral de descodificación, toda descodificación constituye la antesala inminente de una nueva codificación facticia y violenta.

En tal disposición de los términos, Deleuze bosqueja una axiomática general del capitalismo. Como acabamos de explicar, el capitalismo se propone fagocitar todos los flujos deseantes, inscribirlos y registrarlos en absoluto. Pues bien, cuando los flujos deseantes alcanzan el umbral capitalista de descodificación, la recodificación procurará sistemáticamente que tanto el trabajo como la riqueza devengan puros, homogéneos e independientes, de manera que ningún flujo pueda fluir sin estar convenientemente regulado y canalizado en la única dirección del consumo. Por consiguiente, tenemos que la producción es inmediatamente registro y consumo, es decir, el doble movimiento de descodificación/recodificación tiene por objeto llevar el registro y el consumo a la producción $\mathrm{misma}^{42}$. Se trata, no obstante, de un proceso secuencial: en cuanto "producción de producción", el proceso produce la producción de registro y, por su parte, la producción de registro produce la producción de consumo; en el lenguaje del propio autor:

"Una parte de la libido en tanto que energía de producción se ha transformado en energía de registro (Numen), una parte de ésta se transforma en energía de consuma (Voluptas). Esta energía residual es la que anima la tercera síntesis del inconsciente, $[. .$.$] la producción de consumo"43.$

Más allá de las distintas contingencias y de los factores involucrados en estos dos devenires, el capitalismo se constituye por la conjugación abstracta de ambos, proporcionando como resultado un "sujeto-universal" (el consumidor final de nietzscheana memoria) y un "objeto-cualquiera" (la mercancía en sus multíplices formas). La producción del deseo como carencia o "insuficiencia de ser" alcanza su eco en la modalidad de trabajo

capitalistas con la "ley de la tendencia opuesta" de marxiana memoria, según la cual el crecimiento de la masa absoluta de "plusvalía" se produce paralelamente a la baja tendencial de la tasa de ganancia. Cfr. Ibid., p. 41.

${ }^{41}$ La capacidad de la descodificación paranoica para insertar fragmentos en fragmentaciones siempre nuevas comporta una indiferencia del producir y del producto, como revela el concepto de bricolage según el análisis de Lévi-Strauss. Cfr. Claude Lévi-Strauss, La pensé sauvage, París, Plon, 1962 , p. 26 ss.

${ }^{42}$ Cfr. Gilles Deleuze y Félix Guattari, op. cit., pp. 13-14.

${ }^{43}$ Ibid., p. 25. 
más emblemática de nuestro tiempo: el consumption work. Es la eclosión de la sociedad de consumo.

\title{
3. La vigilancia contemporánea: el análisis predictivo o Big Data
}

Una vez dilucidada a grandes rasgos la naturaleza del capitalismo neoliberal desde una perspectiva netamente ontológica, estamos en disposición de indagar la micropolítica que constituye su corolario en la praxis histórico-social, esto es, la cuestión del neoliberalismo en cuanto tecnología. Y es que, si hay nuevo sujeto, es necesario captarlo en las prácticas que producen efectivamente funcionamientos psíquicos de nuevo cuño. Según Foucault, el panóptico no solo sirve para proporcionar sujeción y obediencia sino que también constituye un laboratorio social privilegiado, toda vez que el registro y la clasificación minuciosa de los internos requieren todo un sistema de información, y el panóptico otorga la posibilidad de crear un saber sobre su comportamiento; en palabras del filósofo:

\begin{abstract}
"El Panóptico es un lugar privilegiado para hacer posible la experimentación sobre los hombres, y para analizar con toda certidumbre las transformaciones que se pueden obtener de ellos. [...] El Panóptico funciona como un laboratorio de poder. Gracias a sus mecanismos de observación, gana en eficacia y en capacidad de penetración en el comportamiento de los hombres; un aumento de saber viene a establecerse sobre todas las avanzadas del poder, y descubre objetos que conocer sobre todas las superficies en las que éste viene a ejercerse" 44 .
\end{abstract}

Bajo el claro influjo de Foucault, Lyotard advierte en el texto fundacional de la postmodernidad que, a pesar de experimentar una crisis de legitimidad en el transcurso del siglo XX, el discurso científico sigue ocupando un lugar privilegiado en el orden del saber, abriendo espacios fundamentales para las estrategias industriales y comerciales; en este sentido, observa con mirada visionaria que "en su forma de mercancía informacional indispensable para la potencia productiva, el saber ya es, y lo será aún más, un envite mayor, quizás el más importante, en la competencia mundial por el poder" [la cursiva es nuestra $]^{45}$. Sirvan a modo de ejemplo, entre los múltiples aparatos burocráticos y policiales anexos al antiguo régimen "biopolítico" (dispositivos de "podersaber", como diría Foucault), las estadísticas de la población (conocidas desde antaño como "estadísticas demográficas" o demographics en el mundo de los negocios), unas estadísticas especialmente útiles para salvaguardar y

${ }^{44}$ Michel Foucault, Vigilar y castigar..., op. cit., p. 208.

45 Jean-François Lyotard, La condición postmoderna, Madrid, Cátedra, 1989, p. 17. 
canalizar el proceso de industrialización, en tanto que permiten determinar las especificaciones necesarias para elaborar cabalmente el código normativo que sustenta la sociedad disciplinaria:

"The specifications [...] include age, sex, income level, family composition, urban or rural location, ethnic character, owner-ship of home, automobile, credit card status, social class, and, in the case of hobby and fan magazines, a dedication to photography, model electric trains, sports cars, philately, do-ityourself crafts, foreign travel, kinky sex, etc." ${ }^{46}$.

Sin embargo, como hace notar Han, el dominio de la psique escapa a esta clase de saber científico en absoluto y, por ende, resulta del todo irrelevante en el punto de elaborar un psicoprograma de la población. Pues bien, más allá del debate académico abierto sobre si el panóptico ha sido o no superado de una vez por todas, los nuevos aparatos de vigilancia engendrados por la revolución de las comunicaciones a mediados del Novecientos han venido, si no a sustituir, sí a complementar el dispositivo disciplinario, extendiendo sus dominios del sistema cerrado que le caracteriza a la vida social e íntima de la población. Desde esta perspectiva, el autor surcoreano nos presenta el principal candidato a ocupar el vacío dejado por el panóptico, en una "sociedad de control" (Kontrollgesellschaft) donde la vigilancia forma parte constitutiva de la esfera digital global:

\begin{abstract}
"La demografía no es una psicografía. No explora la psique. En esto reside la diferencia entre la estadística y el Big Data. A partir del Big Data es posible construir no solo el psicoprograma individual, sino también el psicoprograma colectivo, quizás incluso el psicoprograma de lo inconsciente. De este modo sería posible iluminar y explotar a la psique hasta el inconsciente" ${ }^{47}$.
\end{abstract}

Gracias al progreso tecnológico en el campo de la vigilancia, "el historial de cada uno de los usuarios de la red puede revisarse y rastrearse, toda acción telemática deja una huella indeleble y cualquier información puede obtenerse inmediatamente" ${ }^{\prime 4}$. En este contexto, el capitalismo logra la hazaña de penetrar en las capas más profundas de la psique, dejando obsoletas las viejas "estadísticas demográficas" donde se cimentaba la investigación de mercados en la prehistoria digital. Saludada desde numerosos sectores postmodernos

46 Dallas Walker Smythe, Dependency road: communications, capitalism, consciousness, and Canada, Norwood, NH: Ablex, 1981; cfr. Dallas Walker Smythe, "On the audience commodity and its work" [en Meenakshi Gigi Durham y Douglas M. Kellner, eds.: Media and cultural studies: keyworks, Malden, Blackwell, 2006], p. 234.

${ }^{47}$ Cfr. Byung-Chul Han, Psicopolitica..., op. cit., p. 38.

48 Vicente Ordóñez Roig, "De Big Brother a Big Data: reflexiones a propósito de Im Schwarm. Ansichten des Digitalen de Byung-Chul Han”, en Araucaria. Revista Iberoamericana de Filosofia, Politica, Humanidades y Relaciones Internacionales, 20, 40 (2018), p. 764. 
como el reino de la libertad ilimitada ${ }^{49}$, la red mundial de información, las computadoras electrónicas y los dispositivos telemáticos que la conforman materialmente se han revelado, en efecto, como los mejores aliados del sistema capitalista, hasta el prurito de erigirse como la esfera "psicopolítica" por excelencia. Más allá de las clásicas tecnologías unidireccionales (1.0), como la televisión o la radio, el panóptico digital se vale de novedosas tecnologías para convertir la vida cotidiana en un objeto constante de la vigilancia (web 2.0, computadoras, teléfonos móviles, tabletas, relojes inteligentes, etc.), llegando a vislumbrar hasta los detalles más insignificantes de nuestra existencia. Como denuncia Bauman, las nuevas tecnologías de la información se adueñan progresivamente de los espacios abiertos y públicos, de manera tal que la vigilancia se difumina, es decir, se vuelve líquida o gaseosa, está en todas partes y en ninguna ${ }^{50}$. La estructura de la vigilancia cobra así un carácter rizomático, en el sentido de que pivota sobre múltiples aplicaciones e interacciones en el sistema global de comunicación, donde los datos fluyen libremente.

Ahora bien, la donación de los datos no responde a coacciones de ningún tipo, más bien obedece a una necesidad interna; la eficiencia del nuevo panóptico se debe, efectivamente, a la inestimable cooperación de los usuarios:

\footnotetext{
"A los reclusos del panóptico benthamiano se los aislaba con fines disciplinarios y no se les permitía hablar entre ellos. Los residentes del panóptico digital, por el contrario, se comunican intensamente y se desnudan por su propia voluntad. Participan de forma activa en la construcción del panóptico digital" ${ }^{51}$.
}

Para ser exactos, la captura de la información hunde sus raíces en el mecanismo de la confesión. Como refleja la sentencia del Tribunal Constitucional de Alemania sobre el censo nacional de 1984, la autodeterminación informativa ha sido considerada en la historia del siglo XX como un espacio esencial de la libertad y, en este sentido, se constituye como uno de los principales pilares del celebérrimo "Estado del Bienestar": "Serían incompatibles con el derecho a la autodeterminación informativa un orden social y su respectivo orden jurídico en los que el ciudadano no pudiera saber quién sabe de él, así como tampoco qué, cuándo y en qué ocasión se sabe de él"52. Pero como señala Han, hace tiempo que este derecho se ha convertido en un dispositivo neoliberal. Es de recibo reconocerlo: si la privacidad está abandonando progresivamente la esfera de lo íntimo hasta confundirse con lo público, es porque se impone con fuerza una suerte de deseo compulsivo por afirmar la "autenticidad del

${ }^{49}$ El primer eslogan publicitario de Microsoft, Where do you want to go today?, expresa esta tendencia ideológica de manera fehaciente. Cfr. Ibid., p. 21.

${ }^{50}$ Cfr. Zygmunt Bauman y David Lyon, Vigilancia líquida, Barcelona, Paidós, 2013, p. 10.

51 Byung- Chul Han, Psicopolitica..., op. cit., p. 21.

${ }^{52}$ Ibid., p. 24.

Araucaria. Revista Iberoamericana de Filosofía, Política, Humanidades y Relaciones Internacionales, año 22, $\mathrm{n}^{\circ} 43$. Primer semestre de 2020. Pp. 55-76. ISSN 1575-6823 e-ISSN 2340-2199 https://dx.doi.org/10.12795/araucaria.2020.i43.03 
yo" 53 , una pasión desaforada, narcisista e individualista por "ser visto" "54, una "exhibición pornográfica de la intimidad y la esfera privada" ${ }^{55}$ que transgrede impunemente el significado moral del "respeto" (del latín, respectus), alimentando profusamente las bases de datos de la vigilancia contemporánea. En este sentido, Bauman concluye que vivimos en una "sociedad confesional":

"[Una] sociedad en la que se elimina la frontera que antes separaba lo privado de lo público, que convierte la exposición pública de lo privado en una virtud pública y en una obligación, y también que excluye de la comunicación pública a cualquiera que se resista a ser reducido a sus confidencias privadas, junto con todos aquellos que se niegan a hacer confidencias" ${ }^{56}$.

Con el objeto de acelerar la circulación de la información, el dispositivo de la trasparencia desinterioriza a las personas, conduciendo a una exterioridad total: "Nos exige compartir, participar, comunicar nuestras opiniones, necesidades, deseos y preferencias; esto es, contar nuestra vida" ${ }^{57}$.

Desde esta perspectiva, la incipiente "dictadura de la trasparencia", las dinámicas del monitoreo sistemático y la tendencia a la autoexposición pivotan esencialmente, huelga decirlo, sobre las redes sociales y la comunicación digital. Utilizadas primordialmente para el entretenimiento y la diversión, es incuestionable que las facilidades que suponen la masificación de Internet y el uso extensivo de redes sociales para la interacción y la comunicación favorecen ostensiblemente la penetración de la vigilancia en los confines que delimitaban la vida privada de los individuos. Como advierte Han, "el Big Brother digital traspasa su trabajo a los reclusos" $" 58$. Ahora bien, ¿cómo debemos entender dicho "traspaso de poderes" en nuestro mundo neo-orwelliano? Íntimamente vinculado al "trabajo consumidor", el concepto dominante de trabajo en el denominado "capitalismo de plataforma" es, bajo nuestro punto de vista, el "trabajo digital": somos trabajadores de las plataformas digitales. En términos clásicos, el trabajo puede ser definido como todo aquello que hacemos por lo cual recibimos un pago (sueldo, salario, etc.). Además, el trabajo suele estar asociado a un oficio y a un lugar determinado, ya sea una fábrica, una oficina o una tienda, y se identifica generalmente con una actividad preferiblemente evitable, como algo desagradable, alienante o frustrante. Pues bien, el

\footnotetext{
${ }^{53}$ Cfr. Zygmunt Bauman y David Lyon, op. cit., p. 36.

54 El autor español J. Choza señala la erradicación social del pudor como el síntoma principal de semejante desenfreno hedonístico y como signo de nuestro tiempo. Entendido como protección de la intimidad, el menoscabo del pudor se pone de manifiesto en tres ámbitos de la vida cotidiana: la vivienda, el vestido y el lenguaje. Cfr. Jacinto Choza Armenta, La supresión del pudor y otros ensayos, Navarra, EUNSA, 1990.

${ }_{55}$ Byung-Chul Han, Im Schwarm. Ansichten des Digitalen, Berlín, Matthes \& Seitz, 2013, p. 8.

56 Zygmunt Bauman y David Lyon, op. cit., p. 38.

57 Byung- Chul Han, Psicopolitica..., op. cit., p. 29.

58 Ibid., p. 21.
} 
"trabajo digital", por el contrario, aparece como un trabajo productivo pero no remunerado, se desarrolla especialmente en las nuevas redes digitales y, sorprendentemente, aceptamos sus consignas de forma voluntaria, hasta el prurito de que "el miedo a ser observado ha sido vencido por la alegría de ser noticia" 59 . Ligado indisociablemente a valores tan apreciados como la libertad de expresión y la sociabilidad, la diversión o el entretenimiento ${ }^{60}$, el "trabajo digital" comporta, incluso, una variante novedosa del "fetichismo de la mercancía" de marxiana memoria ${ }^{61}$ : el "fetichismo de la mercancía digital"62.

A partir de la información cosechada por el sinóptico digital (esto es, del conjunto de datos o "metadata" que revelan nuestras interacciones en la red), la "data vigilancia" trata de apoderarse del futuro para diseñarlo y controlarlo cual demiurgo arbitrario, estratificando las poblaciones y segmentando los mercados con el objeto de optimizar la oferta de los distintos bienes y servicios. En aras del progreso alcanzado en el procesamiento y almacenamiento de la información a lo largo del Novecientos, la analítica predictiva o Big Data encuentra correlaciones y patrones de comportamiento allí donde las anticuadas "estadísticas demográficas" buscaban desentrañar la causa de los fenómenos, y elabora una clasificación social sobre la base de la moda, la media o la desviación estándar. Desde este prisma, los sistemas electrónicos de la vigilancia convierten algorítmicamente los datos biográficos de los usuarios en predicciones útiles de su comportamiento, unas predicciones en virtud de las cuales se muestran capaces de determinar directa o indirectamente nuestra experiencia, nuestras oportunidades y nuestras decisiones. De aquí se sigue, como advierte Han, el imperio incontestable del cálculo y la aritmética, hasta el extremo de que "todo lo que no es contable o numerable deja de ser" 63 ; dicho con sus propias palabras: "El orden digital totaliza lo computacional o lo aditivo" ${ }^{64}$.

Contemplada en perspectiva histórica, la tendencia general de la ciencia económica ha sido, más allá de sus diferentes corrientes ideológicas, la de pasar por alto la importancia que tienen los mass media, la publicidad, la audiencia

\footnotetext{
59 Zygmunt Bauman y David Lyon, op. cit., p. 32.

${ }^{60}$ Actualmente, lo lúdico, lo cómico y lo humorístico representan un imperativo social generalizado, en línea directa con el proceso de banalización y desubstancialización postmodernas. Cfr. Gilles Lipovetsky, op. cit., p. 136 ss.

${ }^{61}$ Cfr. Karl Marx, El Capital, op. cit., tomo 1, sección primera, capítulo 1, D: "El carácter fetichista de la mercancía y su secreto".

${ }^{62}$ Cfr. Ursula Huws, Labor in the global digital economy. The cybertariat comes of age, Nueva York, Monthly Review Press, 2014. A propósito, toda técnica de dominación genera objetos de devoción que materializan y estabilizan el dominio, como bien ilustran el smartphone o facebook: "El smartphone no es solo un eficiente aparato de vigilancia, sino también un confesionario móvil. Facebook es la iglesia, la sinagoga global (literalmente, la congregación) de lo digital”. Byung- Chul Han, Psicopolitica..., op. cit., p. 26.

${ }^{63}$ Byung-Chul Han, Im Schwarm ..., op. cit., p. 51.

${ }^{64}$ Ibid., p. 57.
}

Araucaria. Revista Iberoamericana de Filosofia, Política, Humanidades y Relaciones Internacionales, año 22, $\mathrm{n}^{\circ} 43$. Primer semestre de 2020. Pp. 55-76. ISSN 1575-6823 e-ISSN 2340-2199 https://dx.doi.org/10.12795/araucaria.2020.i43.03 
y, en definitiva, lo que D. W. Smythe denomina "Industria de la Conciencia"65 para el monopolio del capitalismo, bajo el discutible prejuicio de que los gastos destinados al marketing no tienen consecuencia alguna en forma de beneficios ${ }^{66}$. No obstante, cabe recordar algunas intuiciones excepcionales meritorias en este terreno. Por ejemplo, algunos autores de la tradición keynesiana se han hecho eco, si bien fugazmente, del "control de la conciencia del consumidor" 67 . A partir de los años 60, economistas burgueses han mostrado cierto interés por el comportamiento de los consumidores, con el empeño nada desdeñable de implantar leyes antimonopolio como telón de fondo. Desde la economía institucional, J. K. Galbraith ha apuntado sobre el "papel orgánico" de la publicidad en el sistema. En la tradición marxista, autores tan variopintos como Gramsci, Althusser, R. Williams, Poulantzas o la Escuela de Frankfurt han denunciado la función manipuladora de los mass media y la publicidad.

Sin embargo, a pesar de la incuestionable sagacidad de estos autores, el mérito de dilucidar ese supuesto "papel orgánico" de la publicidad desde la perspectiva de su dimensión histórico-materialista corresponde, para nosotros, a D. W. Smythe. El autor canadiense lleva a cabo una teoría realista de las comunicaciones, analizando empíricamente el papel que juegan los mass media en el sistema capitalista. Para Smythe, la razón de ser de los mass media se traduce en la producción de una mercancía esencial para el funcionamiento de la sociedad de consumo, hasta el punto de que no podría pensarse sin ella: el audience power ${ }^{68}$. Solo así podemos explicarnos las ingentes sumas de dinero destinadas al marketing y la publicidad desde mediados del siglo XX, a raíz de la expansión fulgurante de las tecnologías de la información y la comunicación (TIC) en la totalidad del orbe y de su fuerte arraigo en los modos de vida. Ahora bien, como es lógico, las empresas no invierten su dinero de manera altruista. Al hilo del susodicho principio de "acumulación originaria", su objetivo primordial consiste en vendernos sus bienes y servicios a cualquier precio. Para ello, lo que las empresas compran a sus anunciantes es, literalmente, su "tiempo de atención". Pues bien, aunque Smythe se refiere exclusivamente a los mass media tradicionales que conoció en vida (televisión, radio, periódicos, revistas, vallas publicitarias, etc.), nos sentimos legitimados para extrapolar sus reflexiones sobre el "poder de la audiencia" al papel desempeñado por el homo digitalis en el "capitalismo de plataforma" contemporáneo, toda vez que nuestro "tiempo de atención" se constituye como la principal materia prima de los nuevos medios digitales para recodificar el deseo humano, alimentando de

${ }^{65}$ Cfr. Dallas Walker Smythe, "On the audience commodity...", op. cit., p. 231.

${ }^{66}$ Cfr. John Kenneth Galbraith, The new industrial state, Boston, Houthton Mifflin, 1967, p. 205.

67 Edward Hastings Chamberlin, The theory of monopolistic competition, Cambridge, Harvard University Press, 1931, pp. 113-114.

${ }^{68}$ Cfr. Dallas Walker Smythe, Dependency road..., op. cit.; cfr. Dallas Walker Smythe, "On the audience commodity...", op. cit. 
este modo nuestra voracidad consumista $\mathrm{y}$, a la postre, perpetuando su dominio ilimitadamente.

Frente al mito de que el mercado omnisciente, omnipotente y autorregulador ha triunfado democráticamente, lo cierto es que hace uso constantemente de la violencia y el choque para reproducirse, una violencia consustancial, no hay que olvidarlo, a la acumulación capitalista. En el empeño por colonizar nuestro "tiempo de atención" mediante las tecnologías de la información y la comunicación, el capitalismo salvaje ( $\sin$ restricciones) se ampara en las prerrogativas estatales para implementar todo tipo de medidas invasivas, entre las cuales cabe destacar, a tenor de su eficacia y su alcance global, una suerte de "bombardeo semiótico" o "hiperestimulación informativa", una forma de violencia extraeconómica cuyas funestas consecuencias se resumen en lo que $\mathrm{N}$. Klein denomina con elocuencia "estado de shock"; según la autora canadiense, el régimen neoliberal se vale del shock para vaciar el alma y desarmar la sociedad, de tal suerte que nos sometemos voluntariamente a su programa ${ }^{69}$.

En el afán por plegar nuestros deseos al imperativo del consumo, los nuevos "mánagers del alma"70 ponen en liza una proliferación excesiva de imágenes que proyectan las consignas del consumismo hasta saturar el imaginario en su totalidad. Se trata, ante todo, de incentivar el automatismo de las compras y concebir productos personalizados, pero también experiencias y formas de vida, pues el objetivo fundamental no es tanto la producción de bienes como la producción de realidad, una realidad desprovista de proyecto y de sentido. Efectivamente, la metamorfosis de las técnicas de vigilancia trae consigo un cambio cualitativo de su propósito principal: si el panóptico uniformaba a sus internos para detectar y corregir las desviaciones de la norma que prescribe el comportamiento correcto, el sinóptico abandona toda pretensión de normalización en aras de otras estrategias de dominación sin parangón en la historia, a saber, la incitación y la seducción hacia un ideal de vida buena y un paradigma del mundo fundados en el consumo; en este sentido, concluye Manjarrés, no solo recibimos "lecciones prácticas sobre cómo evitar castigos y obtener recompensas, sino también qué es deseable ser"

Ciertamente, la profusión lujuriosa de imágenes, productos y servicios, así como las presentaciones caleidoscópicas que adoptan, parecen despojar de toda unidad o intención unificadora al paradigma del mundo impuesto por el capitalismo de consumo. A pesar de su apariencia espontánea y caótica, empero, el proceso de "hiperestimulación semiótica" procede de una racionalidad

${ }^{69}$ Cfr. Naomi Klein, La doctrina del shock. El auge del capitalismo del desastre, Buenos Aires, Paidós, 2015.

${ }^{70}$ La fórmula es de Lacan y ha sido retomada por Valérie Brunel. Cfr. Valérie Brunel, Les Managers de l'âme. Le Développement personnel en entreprise, nouvele pratique de pouvoir?, París, La Découverte, 2004.

${ }^{71}$ Emilio Manjarrés Chavarriaga, Facebook en la sociedad de control: aplicación simultánea del panóptico y del sinóptico en un dispositivo de vigilancia y control, Medellín, EAFIT, 2017, p. 103.

Araucaria. Revista Iberoamericana de Filosofia, Politica, Humanidades y Relaciones Internacionales, año $22, \mathrm{n}^{\circ} 43$. Primer semestre de 2020. Pp. 55-76. ISSN 1575-6823 e-ISSN 2340-2199 https://dx.doi.org/10.12795/araucaria.2020.i43.03 
subterránea, difusa y global, una lógica que define y construye la esencia misma del neoliberalismo. Ante las críticas de explotación y reificación de los individuos, el sistema responde con lenguajes personalizados no ya solo a la hora de consumir sino en todas las esferas de la existencia humana, en lo que constituye una destrucción cool de lo social ${ }^{72}$. La individualización obtenida por los algoritmos de la analítica predictiva permite ajustar minuciosamente la oferta y la demanda, de manera que el mercado ofrece cada vez más opciones y combinaciones a medida, generando un ambiente eufórico de tentación y proximidad. En lugar de sustraer o prohibir, el poder del presente domina agradando y colmando. Su secreto radica en explotar la libertad, convertida en la libre elección entre distintas ofertas. Es un poder sutil y flexible, amable e inteligente.

\section{Referencias bibliográficas:}

Alberto Melucci, Acción colectiva, vida cotidiana y democracia, Ciudad de México, Colegio de México, 1999.

Andrea Fagioli, "Acumulación originaria y capitalismo neoliberal. Una posible lectura del Chile post-golpe”, en Isegoría. Revista de Filosofía Moral y Política, 59 (2018).

Aristóteles, De Republica, Buenos Aires, Losada, 2005.

Borja García Ferrer, "A vueltas con Heidegger. La filosofía como terapia frente al "nihilismo tecnológico"” [en Luis Sáez Rueda, ed.: El malestar de Occidente. Perspectivas filosóficas sobre una civilización enferma, Anchor Academic Publishing, Hamburg, 2017].

Bruno Astarian, Las huelgas en Francia durante mayo y junio de 1968, Madrid, Traficantes de Sueños, 2008.

Byung-Chul Han, Im Schwarm. Ansichten des Digitalen, Berlín, Matthes \& Seitz, 2013.

Byung-Chul Han, Psicopolítica. Neoliberalismo y nuevas técnicas de poder, Barcelona, Herder, 2014.

Christian Laval y Pierre Dardot, La nueva razón del mundo. Ensayo sobre la sociedad neoliberal, Barcelona, Gedisa, 2013.

Claude Lévi-Strauss, La pensé sauvage, París, Plon, 1962.

Dallas Walker Smythe, Dependency road: communications, capitalism, consciousness Canada, Norwood, NH: Ablex, 1981.

Dallas Walker Smythe, "On the audience commodity and its work" [en Meenakshi Gigi Durham y Douglas M. Kellner, eds.: Media and cultural studies: keyworks, Malden, Blackwell, 2006].

${ }^{72}$ Cfr. Gilles Lipovetsky, op. cit., pp. 18-25.

Araucaria. Revista Iberoamericana de Filosofia, Política, Humanidades y Relaciones Internacionales, año $22, \mathrm{n}^{\circ} 43$. Primer semestre de 2020. Pp. 55-76. ISSN 1575-6823 e-ISSN 2340-2199 https://dx.doi.org/10.12795/araucaria.2020.i43.03 
David Harvey, Guía de El Capital de Marx. Libro Primero, Madrid, Akal, 2014. David Lyon, "The search for surveillance theroies" [en Theorizing surveillance: the panopticon and beyond, Cullompton, Willan Publishing, 2006].

Diego Sánchez Meca, El nihilismo. Perspectivas sobre la historia espiritual de Europa, Madrid, Síntesis, 2004.

Edward Hastings Chamberlin, The theory of monopolistic competition, Cambridge, Harvard University Press, 1931.

Emilio Manjarrés Chavarriaga, Facebook en la sociedad de control: aplicación simultánea del panóptico y del sinóptico en un dispositivo de vigilancia y control, Medellín, EAFIT, 2017.

Friedrich Nietzsche, Crepúsculo de los ídolos, Madrid, Alianza, 1980.

Gilles Deleuze, "Post-scriptum sobre las sociedades de control" [en Conversaciones 1972-1990, Valencia, Pre-Textos, 1999].

Gilles Deleuze y Félix Guattari, El Anti Edipo. Capitalismo y esquizofrenia, Barcelona, Paidós, 1985.

Guy Standing, El precariado, Barcelona, Pasado y Presente, 2013.

Herbert Marcuse, Eros y Civilización, Barcelona, Seix Barral, 1976.

Jacinto Choza Armenta, La supresión del pudor y otros ensayos, Navarra, EUNSA, 1990.

Jean-François Lyotard, La condición postmoderna, Madrid, Cátedra, 1989.

John Kenneth Galbraith, The new industrial state, Boston, Houthton Mifflin, 1967.

Karl Marx, El Capital, México, Siglo XXI, 1975.

Kathryn Wheeler y Miriam Glucksmann, Household recycling and consumption work: social and moral economies, Londres, Palgrave McMillan, 2015.

Martin Heidegger, "La superación de la metafísica" [en Eustaquio Barjau, trad.: Conferencias y artículos, Barcelona, Serbal, 1994].

Maurizio Lazzarato, La fábrica del hombre endeudado. Ensayo sobre la condición neoliberal, Buenos Aires, Amorrortu, 2013.

Michel Foucault, Vigilar y castigar. Nacimiento de la prisión, Buenos Aires, Siglo XXI, 2002.

Michel Foucault, Historia de la sexualidad I. La voluntad de saber, Madrid, Siglo XXI, 2006.

Naomi Klein, La doctrina del shock. El auge del capitalismo del desastre, Buenos Aires, Paidós, 2015.

Paolo Virno, Gramática de la multitud: para un análisis de las formas de vida contemporáneas, Madrid, Traficantes de sueños, 2003.

Roberto Esposito, Bíos. Biopolítica y filosofía, Buenos Aires, Amorrortu, 2006. Ronald Butt, "Mrs Thatcher: The First Two Years", en The Sunday Times, 3 de mayo de 1981 . 
Rosa Luxemburgo, La acumulación del capital, Madrid, Ediciones Internacionales Sedov, 2011.

Samir Amin, La acumulación en escala mundial, Buenos Aires, Siglo XXI, 1975.

Sergio Bologna y Giairo Daghini, Maggio '68 in Francia, Roma, DeriveApprodi, 2008.

Thomas Pikkety, El capital en el siglo XXI, Madrid, Fondo de Cultura Económica, 2014.

Ursula Huws, Labor in the global digital economy. The cybertariat comes of age, Nueva York, Monthly Review Press, 2014.

Valérie Brunel, Les Managers de l'âme. Le Développement personnel en entreprise, nouvele pratique de pouvoir?, París, La Découverte, 2004.

Vicente Ordóñez Roig, "De Big Brother a Big Data: reflexiones a propósito de Im Schwarm. Ansichten des Digitalen de Byung-Chul Han", en Araucaria. Revista Iberoamericana de Filosofía, Política, Humanidades y Relaciones Internacionales, 20, 40 (2018).

Walter Lippmann, The Good Society, Boston, Little, Brown and Company, 1937.

William Bogard, "Surveillance assemblages and lines of flight" [en Theorizing surveillance: the panopticon and beyond, Cullompton, Willan Publishing, 2006].

Zygmunt Bauman y David Lyon, Vigilancia líquida, Barcelona, Paidós, 2013. 